\title{
VIN usual type-from the past to the future
}

\section{Mario Preti ${ }^{1^{*}}$, Sarah Igidbashian ${ }^{1}$, Silvano Costa ${ }^{2^{*}}$, Paolo Cristoforoni ${ }^{3^{*}}$, Luciano Mariani ${ }^{*}$, Massimo Origoni ${ }^{5^{*}}$, Maria T Sandri ${ }^{*}$, Sara Boveri ${ }^{1}$, Noemi Spolti ${ }^{1}$, Laura Spinaci ${ }^{1}$, Francesca Sanvito ${ }^{1}$, Eleonora P Preti ${ }^{1}$, Adriana Falasca ${ }^{1}$, Gianluigi Radici ${ }^{1}$ and Leonardo Micheletti ${ }^{7}$}

${ }^{1}$ Preventive Gynecology Unit, European Institute of Oncology, Milano 20100, Italy

${ }^{2}$ M.F. Toniolo Hospital, Bologna 40100, Italy

${ }^{3}$ Villa Montallegro, Genova 16100 , Italy

${ }^{4} \mathrm{HPV}$-Unit Gynecologic Oncology, Regina Elena National Cancer Institute of Rome, Rome 00100, Italy

${ }^{5}$ Department of Obstetrics and Gynecology, Vita Salute San Raffaele University School of Medicine, Milano 20100, Italy

${ }^{6}$ Division of Laboratory Medicine, European Institute of Oncology, Milano 20100, Italy

${ }^{7}$ Department of Obstetrics and Gynecology, University of Torino, Torino 10100, Italy

*The Italian HPV Study Group (IHSG)

Correspondence to: Mario Preti. Email: Mario.preti@ieo.it

\section{Abstract}

Usual vulvar intraepithelial neoplasia ( $\mathrm{UVIN}$ ) is the most common VIN type, generally related to a human papillomavirus (HPV) infection, predominantly type 16 . The incidence of $\mathrm{UVIN}$ has been increasing over the last decades, and a bimodal peak is observed at the age of 40-44 and over 55 years.

Almost $40 \%$ of patients with uVIN have a past, concomitant or future HPV-associated lesion of the lower genital tract. HPV-related malignancies are associated with a persistent HPV infection. The host immune response is of crucial importance in determining clearance or persistence of both HPV infections and HPV-related VIN. About $60 \%$ of the patients present with symptoms. Clinical features of uVIN vary in site, number, size, shape, colour, and thickness of lesions. Multicentric disease is often present.

Most uVIN lesions are positive at immunohistochemistry to $\mathrm{p} 16^{\text {ink4a }}$ and $\mathrm{p} 14^{\text {arf }}$, but negative to p53.

Published: 29/04/2015

Received: $10 / 12 / 2014$

ecancer 2015, 9:531 DOI: 10.3332/ecancer.2015.531

Copyright: (c) the authors; licensee ecancermedicalscience. This is an Open Access article distributed under the terms of the Creative Commons Attribution License (http://creativecommons.org/licenses/by/3.0), which permits unrestricted use, distribution, and reproduction in any medium, provided the original work is properly cited. 
Irrespective of surgical treatment used, uVIN recurrence rates are high. Positive margins do not predict the development of invasive disease and the need to re-excide the tissue around the scare remains to be demonstrated. Therefore, considering the low progression rate of uVIN and psycosexual sequelae, treatments should be as conservative as possible.

Medical treatments available are mainly based on immunotherapy to induce normalisation of immune cell count in uVIN. None are approved by the food and drug administration (FDA) for the treatment of UVIN. If medical treatment is performed, adequate biopsies are required to reduce the risk of unrecognised invasive disease. Some studies suggest that failure to respond to immunotherapy might be related to a local immunosuppressive microenvironment, but knowledge of the UVIN microenvironment is limited. Moreover, our knowledge of the potential mechanisms involved in the escape of HPV-induced lesions from the immune system has many gaps.

HPV vaccines have been demonstrated to be effective in preventing UVIN, with $94.9 \%$ efficacy in the HPV-naive population, while studies on therapeutic vaccines are limited. The low incidence of VIN requires large multicentre studies to determine the best way to manage affected patients and to investigate the immunological characteristics of the 'vulvar microenviroment' which leads to the persistence of HPV.

Keywords: VIN, vulvar intraepithelial neoplasia, HPV infection

\section{Classification}

The definition of vulvar intraepithelial neoplasia (VIN) refers to a proliferation of atypical basal cells in the vulvar epithelium. The acronym VIN was introduced at the beginning of the 1980s to define with a single term dysplastic lesions and carcinoma in situ of the vulva. Until then, these lesions were identified with different terms such as erythroplasia of Queyrat, Bowen's disease, and hyperplastic dystrophy with atypia. The VIN classification was adopted in 1986 by the International Society for the Study of Vulvar Disease (ISSVD) including intraepithelial neoplasia of the squamous epithelium (divided into three grades based on the thickness of the epithelium affected by cellular atypia) and non-squamous VIN (Paget's disease and vulvar melanoma in situ) [1].

There has been criticism of the 1986 ISSVD VIN classification. The first point of criticism was inclusion in the classification of Paget's disease and vulvar melanoma in situ (not squamous intraepithelial neoplasia) that have different epidemiology, risk factors, and natural history compared to squamous VIN.

Other criticisms were as follows:

- Grading of VIN was similar to cervical intraepithelial neoplasia classification (CIN) despite the origin of VIN from a mature squamous skin/mucous epithelium and CIN from a transition epithelium.

- Clinical evidence that VIN1 does not represent a precancerous lesion but is just a sign of an HPV infection, a regressive alteration or an inflammatory disease.

- Reproducibility studies on the histopathological diagnosis of VIN that showed a very low reproducibility of VIN1 and a distinction between HPV-related VIN and non-HPV-related VIN

Therefore, in 2004, the ISSVD introduced a new classification for VIN on the basis of morphologic and pathogenetic criteria [2].

In this new classification, the diagnostic category of VIN 1 has been abolished, and histological changes previously included under the term VIN 1 are described as condyloma and/or HPV effects. The term VIN has been reserved only to the lesions previously classified as VIN 2 and 3 and differentiated VIN.

Three diagnostic terms were introduced to classify the specific pathogenetic VIN types as follows:

- VIN usual type (uVIN)

- VIN differentiated type (dVIN)

- VIN unclassified type (VIN NOS)

Usual type VIN (bowenoid, basaloid, and mixed) is the most common VIN type and is generally related to a human papillomavirus (HPV) 16 infection [3], and it occurs predominantly in younger women and tends to be multifocal.

Differentiated VIN is less common (2-10\% of all reported VIN) and generally not related to HPV, it is mostly unifocal and usually found in older women with associated chronic dermatoses as lichen sclerosus. 


\section{Epidemiology}

The worldwide incidence of UVIN in young women has been increasing over recent decades [4-8]. From 1992 to 2005, the incidence of UVIN has almost doubled from $1.2 / 100,000$ to $2.1 / 100,000$, while the incidence of invasive vulvar cancer (IVC) remained stable. The increase in awareness of vulvar premalignant lesions may have led to the observed increase in VIN incidence, but also it may result in the removal of UVIN before these become invasive. A bimodal peak incidence of uVIN was observed at the age of $40-44$ years and over 55 years $[5,7,8]$. A past, concomitant or future HPV-associated lesion of the lower genital tract is shown in $41 \%$ of patients with uVIN [8], with younger patients being at a higher risk of multifocal lesions. Van de Nieuwenhof et al showed that the risk of a subsequent diagnosis of vulvar squamous cell carcinoma (SCC) significantly increased with the age of diagnosis of uVIN, beginning with $2.7 \%$ for the age group 15-29 and increased to $8.5 \%$ for the group $>75$ years of age. In addition, they showed that the time between diagnosis of uVIN and SCC significantly shortened with increasing age, that is, 50 months for the age group of 15-29 years and 25 months for the age group of more than 75 years [8].

\section{Pathogenesis}

The recent analysis of over 2,000 intraepithelial and invasive lesions of the vulva made by the HPV VVAP study group detected HPV-DNA in $86.7 \%$ of the VIN cases [9]. The low proportion of HPV-negative lesions correlates with the lower proportion of dVIN compared to uVIN. HPV 16 was the commonest genotype (77.3\%) detected in uVIN, followed by HPV $33(10.6 \%)$ and HPV18 (2.5\%). In contrast to the high proportion of HPV-positive VIN, only $28.6 \%$ of IVC were HPV-DNA positive [9]. It is well known that IVC not related to HPV are more frequent than those related to HPV. These carcinomas are usually found in patients affected with lichen sclerosus or lichen planus and derived from dVIN.

The malignant potential of UVIN is difficult to assess being usually surgically removed to prevent invasive disease. A review of 3,322 treated and untreated VIN showed a progression to IVC in $9 \%$ of the untreated cases within 1-8 years, while in case of treated uVIN, the malignant potential was 3.3\% [10]. The risk of future IVC in VIN-treated patients is considered to be around 3-4\% [10-13].

Similar to the intraepithelial neoplasia found in IVC, the most common HPV type detected is HPV 16 (72.5\%), followed by HPV 33 (6.5\%) and HPV 18 (4.6\%). While HPV 45 was almost not present in VIN, it was the fourth most common HPV (3.3\%) in IVC showing a possible different contribution of this HPV genotype to HPV-associated invasion in the vulva [9].

HPV-related malignancies are associated with a persistent HPV infection. In $90 \%$ of cases, the immune system is capable of clearing a transient HPV infection within 2 years [14]. The host immune response is of crucial importance in determining clearance or persistence of both HPV infections and HPV-related VIN [14-19]. This is clearly seen in immune-compromised patients where the malignant potential of UVIN is 50-fold higher compared to the general population [20]. Failure of the immune system to produce an effective response to high-risk HPV is related to both viral persistence and various host factors [21]. HPV produces several proteins among which there are the oncoproteins E6 and E7. The longer the infection persists, the longer E6 and E7 can interfere with important control mechanisms of the cell cycle [22-26]. HPV E6 can lead to the dysfunction of the tumour suppressor gene p53 [27], while E7 can inactivate the retinoblastoma tumour suppressor gene (pRb), which leads to the overexpression of $\mathrm{p} 16^{\text {ink4a }}$ and $\mathrm{p} 14^{\text {arf }}$ and hyperproliferation of infected cells [24, 28]. Most uVIN lesions are positive at immunohistochemistry to $\mathrm{p} 16^{\text {ink4a }}$ and p14 arf, but negative to p53 [24-26, 29, 30]. The knowledge of the role of these defined tumour-specific antigens, E6 and E7, represents an excellent basis for the development of strategies aiming to reinforce the immune response to prevent HPV-related cancers.

\section{Clinical features and diagnosis}

Screening tests for the diagnosis of VIN are not available: uVIN is diagnosed during the visual assessment of the vulva. To confirm the diagnosis, a biopsy of suspicious lesions should be performed using local anesthesia. The optimal biopsy is a punch or a small incision biopsy taken from the edge of the lesion with the inclusion of a piece of surrounding normal tissue [31]. 
UVIN can be asymptomatic, diagnosed during routine gynaecological visits, in patients with abnormal pap smear or positive HPV cervical test, or symptomatic in about $60 \%$ of the patients [10, 32].

The clinical features of UVIN vary in site anywhere on the vulva, number, size, shape, colour, and thickness of lesions. uVIN are usually multifocal, located around the introitus often involving the labia minora, and elevated. Lesions are sharply defined although the outline may be irregular or serpiginous [33] (Figures 1-5).

Multicentric disease (lesions of cervix, vagina, or anus) is often present in cases with uVIN, underlining the 'HPV field effect' that involves squamous epithelium from cervix to perianal area and the need of accurate examination in uVIN-affected patients. Therefore, a careful examination of all the vulva, perineum, perianal areas and comprehending also the cervix and vagina is mandatory.

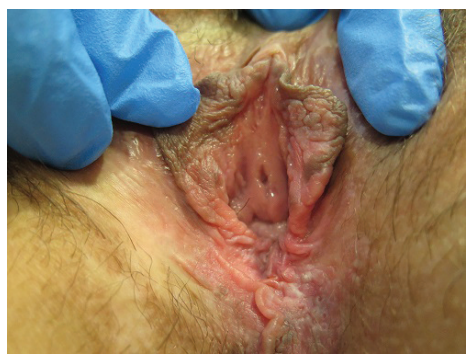

Figure 1. Twenty-nine-year-old patient with uVIN. Vulvar multifocal lesions: white and brown plaque on the inner surface of the left labium minus; white and brown papules on the perineum.

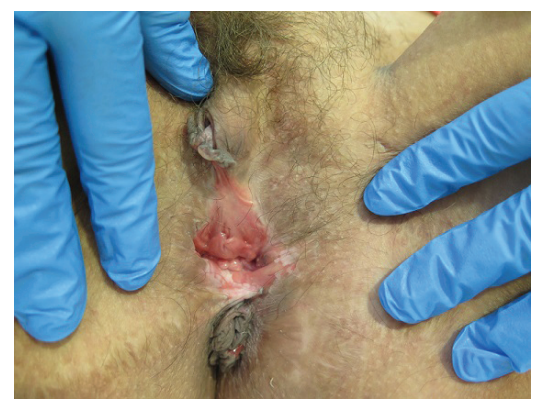

Figure 2. Thirty-eight-year-old patient with uVIN. Confluent white plaques on the posterior vaginal vestibule after partial simple vulvectomy for superficially invasive keratinising squamous cell carcinoma.

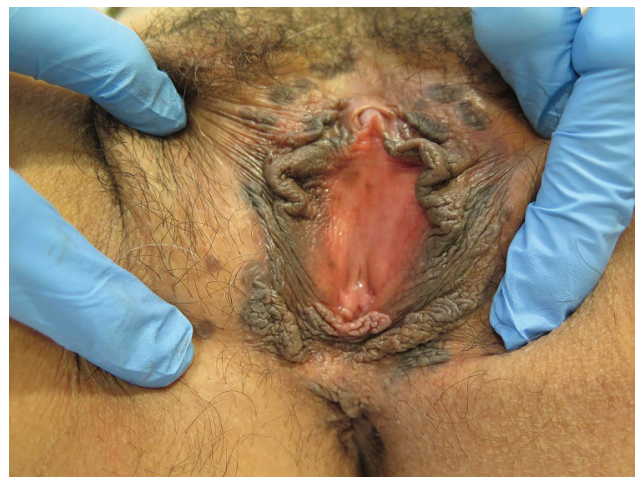

Figure 3. Thirty-nine-year-old patient with uVIN. Multifocal bilateral brown papules on the labia majora from the anterior part of vulva until the perineum. 
ecancer 2015, 9:531

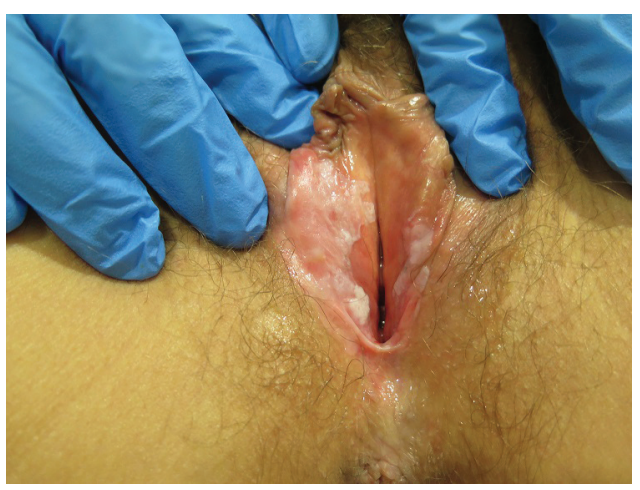

Figure 4. Fifty-four-year-old patient with uVIN. White papule on the inner surface of the posterior right labium minus in the context of confluent bilateral white macules on the vaginal vestibule.

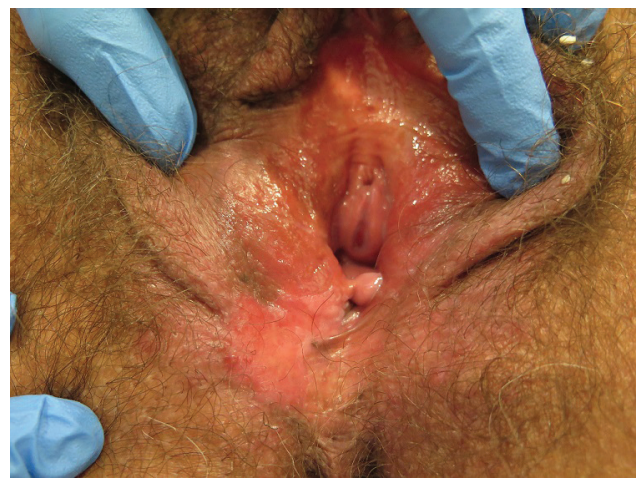

Figure 5. Fifty-nine-year-old patient with uVIN. Unifocal white, red, and brownish plaque on the right posterior part of the vulva, in the context of diffuse vulvar erythema, with excoriations caused by scratching.

\section{Treatment}

The ideal approach to uVIN is to ensure the absence of stromal invasion, improve subjective symptoms, and reduce the risk of recurrence. Unfortunately, the surgical removal of the lesion represents, for most cases, the current standard approach to uVIN [33].

Indeed, no clinical appearance helps in distinguishing uVIN with stromal invasion from those without invasion, and in 12-17\% of women undergoing VIN excision, a clinically unrecognised invasion is histologically diagnosed [11, 38-40]. In the past, extensive surgery has been performed to achieve this goal, leading in many cases to the disruption of both vulvar anatomy and function. More limited surgery is now being used in order to alter morphology as little as possible and to preserve vulvar function. Irrespective of the surgical treatment used recurrence rates are high $[10,11,34]$. Considering the low progression rate of UVIN and the young age of affected women treatments should be as conservative as possible with minimal effects on the psycosexual sphere and quality of life (QoL) of these women.

\section{Surgical treatment}

Local excision removing all visible lesions can be performed with different techniques: scalpel, electrosurgery, or laser. All techniques seem to have a similar efficacy. 
Surgery is still considered the treatment of choice, but surgeons should be as conservative as possible to reduce changes in the QoL of treated patients [33]. Optimal aesthetic and functional perineovulvar reconstruction is now considered as an integral part of the treatment of these lesions. Recurrence rate after excision ranges from $20 \%$ to $40 \%$ [12]. The presence of multifocal disease is associated with a higher incidence of recurrence [33, 34].

There are no definitive studies evaluating the safety margins in VIN resections, but recent papers demonstrated that positive margins do not predict the development of invasive disease [35]. In other words, when the pathological specimen describes involved margins and no stromal invasion is noted, for the most part of cases, it is enough to follow patient without re-exciding the tissue around the scare. Ongoing studies on adjuvant therapy with imiquimod in these cases are reported in next paragraph.

Carbon dioxide $\left(\mathrm{CO}_{2}\right)$ laser excision yields a good cosmetic and functional result in experienced hands and gives a surgical specimen for histologic evaluation which is not present in case of $\mathrm{CO}_{2}$ laser vapourisation. Therefore, when $\mathrm{CO}_{2}$ laser vapourisation is planned, multiple biopsies should be performed to rule out invasion. Recurrence-free survival is lower in case of laser-vapourisation, probably because this technique is more often used in multifocal and multicentric lesions [11, 12, 36, 37].

\section{Medical treatment}

Many medical treatments have been introduced to avoid or limit surgery in patients with UVIN. Most treatments lack of enough evidence because of the few number of subjects studied, of the different inclusion criteria, comparison groups or follow-up used in the studies [28]. Therefore, no medications are approved by the FDA for UVIN treatment.

Moreover, considering the high number of clinically unrecognised invasion found in the histology examination of VIN excisions, if surgical treatment is not performed, adequate biopsies are required to reduce the risk of unrecognised invasive disease.

Medical treatments available are mainly based on immunotherapy. The different forms of immunotherapies that have been developed and tested for the treatment of UVIN are aimed at overcoming the 'inertia' of the immune system in these patients.

\section{Imiquimod}

Imiquimod, 1-(2-methylpropyl)-1H-imidazo[4, 5-c]quinolin-4-amine (also known as R-837 and S-26308), is a non-nucleoside heterocyclic amine that belongs to immune-response modifiers [41]. In vivo studies have demonstrated that imiquimod is a potent inducer of interferon (INF) $\alpha$, tumour necrosis factor $\alpha$, and interleukin-6 [42-44]. In animal models, imiquimod has demonstrated potent antiviral and antitumour effects [41].

Imiquimod $5 \%$ is licensed for the treatment of external genital warts, actinic keratosis, and basal cell carcinoma. Imiquimod $5 \%$ acts through a toll-like receptor (TLR7) inducing T cells activation and proinflammatory cytokines release $[45,46]$. A thin layer of imiquimod $5 \%$ cream is applied on the lesion two or three times per week for a period of 12-16 weeks. Side effects are mainly local inflammatory reactions, itching, burning, and flu-like signs. In case of severe side effects, application can be reduced to once a week or stopped for a week.

The largest prospective, randomised, double-blind, placebo-controlled study analysed 52 cases, of which $35 \%$ had a complete response and $46 \%$ a partial response [47]. This study showed an effective long term efficacy of imiquimod, since VIN recurred in only one case of the complete respondents [48]. A recent meta-analysis that assessed 104 patients confirms the effectiveness of the treatment [49].

Imiquimod-induced clearance of HPV was associated with the normalisation of immune cell count in UVIN [48]. Many different pre-existing conditions can determine failure of patients' responsiveness to immunotherapy: lesion size, lack of immune infiltration, CD4+ or CD8+ HPV-specific T-cell response, defects in HLA class I expression, IFNY-associated response, genes of antigen presentation pathway, genes involved in T-cell migration and in chemo or cytokine production [21].

Imiquimod 5\% has also been tested after surgery in comparison to surgery alone. The results in 80 patients demonstrated that during 5 years of follow-up, the overall complete response, the recurrence rate, and the disease-free time were similar in the two treatment options [50]. 


\section{Therapeutic vaccines}

Therapeutic vaccines are designed to reinforce HPV-specific CD4+ and CD8+ T-cell responses in uVIN lesions [51]. Most vaccines elicit a specific immunity against HPV E6 and E7 proteins [52].

Two studies investigated the effectiveness of a combination of TA-HPV, a vaccinia virus encoding HPV16/18 E6/E7, and TA-CIN, an HPV 16 L2 E6/E7 fusion protein, in a total of 39 women with HPV 16 high-grade VIN. A reduction in lesion size was seen in 9 patients, while 25 remained stable and 5 progressed. This regimen proved to be immunogenic but no relation between induction of HPV 16-specific immunity and clinical outcome was observed $[53,54]$.

Kenter et al tested a synthetic long peptide (SLP) vaccine in 20 patients with HPV16-positive high-grade VIN. At 12 months of follow-up, 15 patients showed clinical response with a complete response in 9 cases. The complete response was maintained at 24 months of follow-up. In this trial, vaccine-induced T-cell responses were seen in all patients and complete responders had a significantly stronger response of IFNY-associated proliferative CD4+ T cells and a broad response of CD8+ IFNy T cells [55].

Small trials showed results with therapeutic vaccines; however, the costs for the development of vaccines are high, and at the moment, the focus is on preventive vaccines rather than therapies.

\section{Photodynamic therapy}

Photodynamic therapy (PDT) uses a tumour-localising photosensitiser, 5-aminolevulinic acid, in combination with non-thermal light to generate tumour-directed cell death and induce local inflammation, which activates antigen-presenting cells (APCs) and induces effector T cells. Several non-randomised and uncontrolled studies were conducted on UVIN with response rates varying from $0 \%$ to $71 \%$, with unifocal lesions responding better than multifocal, pigmented, and high-grade lesions [56-60]. Recurrence rate (around 48\%) was similar to laser vapourisation and surgery [12]. Data on PDT are limited to small case series with variable response rates; patient tolerance of side effects, such as pain, could limit its use and costs are high.

\section{Known and unknown of immunological microenvironment}

It has long been known that the adaptive immune response, in particular T cells, confer protection against HPV-induced diseases [61, 62]. Overall, HPV-induced diseases are associated with a lack of a strong HPV-specific CD4+ and CD8+ T cell [63]. The medical treatments available for UVIN are all associated with an increase in intralesional CD4+ and CD8+ T cells. Unfortunately, the available immunotherapeutic approaches often fail to induce clinical responses. Some studies suggest that failure to respond to immunotherapy might be related to a local immunosuppressive microenvironment, but knowledge on uVIN microenvironment is limited [19, 46, 48, 64-66]. Moreover, the knowledge of the potential mechanisms involved in the escape of HPV-induced lesions from the immune system has many gaps.

Winters et al found that initial not-responders to imiquimod seemed to be relatively refractory, probably because of their unfavourable local immune environment, in particular, the increased proportions of T regulatory cells (Tregs) [65]. Moreover, patients with lesions unresponsive to therapeutic vaccines showed a reduced systemic vaccine response but also increased number of lesions associated with immune suppressive Tregs [55, 66, 67]. Failure to cure some HPV-related premalignant and malignant lesions appears to result from an unfavourable balance in effector T cells and Tregs. Success of immunotherapies might need to address the means to alter this balance [68].

Spontaneous regression and clearance of HPV is associated with systemic HPV-specific CD4+ and CD8+ T-cell responses and normalisation of immune cell infiltration [62, 68]. The UVIN microenvironment is characterised by a dermal immune activity, with an influx of mature dendritic cells (DCs), natural killer cells (NK), and both CD4+ and CD8+ T cells, while the epidermis shows a decreased number of CD8+ T cells and increase in immature DCs and Langerhans cells (LCs) [19, 48, 56]. 
Moreover, van Esch et al showed an alteration in human leukocyte antigen (HLA) molecules not only in HPV-induced cancers but even in the premalignant stage. They detected a partial downregulation of HLA-class I in more than $70 \%$ of uVIN lesions and in $80 \%$ of HPV-related IVC. However, the downregulation of HLA class I expression in the majority of UVIN was not associated with the loss of heterozigosity (LOH) in contrast with the findings in IVC. This downregulation in UVIN was considered 'soft wired' since the expression can be restored applying some changes to the microenvironment, like stimulating with IFNY. All patients with uVIN evaluated that showed a good clinical response to HPV 16 SLP vaccine had this partial downregulation in UVIN. In fact, the vaccine-induced HPV 16-specific type helper T cells infiltrating the lesion can provide IFNy in the microenvironment [69].

Immunotherapy may strengthen the pre-existent activation of the local immune system, and immunologically active primary lesions may be more likely to respond to immunotherapy, also having a lower number of regulatory $T$ cells subsets. Therefore, less immunologically active UVIN lesions, recurrent lesions and IVC may represent the harder challenge for immunotherapies [51].

An increased comprehension of the immunological environment that can be found in uVIN might help to better understand the conditions that favour the persistence of HPV and the development of UVIN and its potential oncogenic transformation to IVC. It might also help to define immunological markers that can predict responsiveness to immunotherapy.

\section{Prevention}

HPV prophylactic vaccination has demonstrated to be effective in preventing uVIN. The vaccine efficacy against HPV 16- and/or HPV 18-related VIN is highest in the HPV-naive population, with a 94.9\% efficacy [70]. Although prevention of IVC was not demonstrated, prevention of premalignant UVIN may anticipate a reduction of rates of HPV-related IVC [71].

HPV vaccines have also demonstrated a partial cross-protective efficacy against non-vaccine HPV types, such as HPV 31, 33, and HPV 45 [72-75]. This could lead to an even higher efficacy in reducing the incidence of UVIN and possibly IVC.

Moreover, studies on previous HPV vaccination in women treated for HPV-related diseases of the cervix, vulva, or vagina showed a reduction in subsequent cervical, vulvar, and vaginal intraepithelial neoplasia and genital warts [76].

An estabilished risk factor for vulvar cancer is smoking. Therefore, smoking should be discouraged [77].

\section{Conclusion}

VIN is observed by a wide variety of medical specialists who should be aware of the crucial importance of careful vulvar examination, the malignant potential of the lesion, and the possibilities of conservative treatment depending on VIN localisation, size, focality, associated diseases, age, and psychological conditions of the patient. Long-term surveillance in all treated women is essential as removal of the lesion does not represent a complete prevention of invasive cancer.

The low incidence of VIN requires large multicentre studies to determine the best way to manage affected patients and to investigate the immunological characteristics of the 'vulvar microenvironment' that leads to the persistence of HPV.

\section{Conflicts of interest}

The authors have no conflict of interest

\section{Acknowledgment}

Dedicated to Mario Sideri. He was flying high on the way to prevention. His example, ideas, and his enthusiasm will light our path. 


\section{References}

1. Wilkinson EJ, Kneale BL and Lynch FW (1986) Report of the ISSVD terminology committee: VIN J Reprod Med 31973-974

2. Sideri M et al (2005) Squamous vulvar intraepithelial neoplasia: 2004 modified terminology, ISSVD vulvar oncology subcommittee J Reprod Med 50 807-810

3. De Vuijst $\mathrm{H}$ et al (2009) Prevalence and type distribution of human papillomavirus in carcinoma and intraepithelial neoplasia of the vulva, vagina and anus: a metaanalysis Int J Cancer 124 1626-1636 DOI: 10.1002/ijc.24116

4. Jones RW, Baranyai J and Stables S (1997) Trends in squamous cell carcinoma of the vulva: the influence of vulvar intraepithelial neoplasia Obstet Gynecol 90 448-452 DOI: 10.1016/S0029-7844(97)00298-6 PMID: $\underline{9277660}$

5. Joura EA et al (2000) Trends in vulvar neoplasia. Increasing incidence of vulvar intraepithelial neoplasia and squamous cell carcinoma of the vulva in young women J Reprod Med 45 613-615 PMID: 10986677

6. Judson PL et al (2006) Trends in the incidence of invasive and in situ vulvar carcinoma Obstet Gynecol 107 1018-1022 DOI: 10.1097/01.AOG.0000210268.57527.a1 PMID: 16648405

7. Baandrup $L$ et al (2011) In situ and invasive squamous cell carcinoma of the vulva in Denmark 1978-2007-a nationwide population-based study Gynecol Oncol 122 45-49 DOI: 10.1016/j.ygyno.2011.03.016 PMID: 21474166

8. Van de Nieuwenhof HP et al (2009) Vulvar squamous cell carcinoma development after diagnosis of VIN increases with age Eur J Cancer 45 851-856 DOI: 10.1016/j.ejca.2008.11.037 PMID: 19117749

9. De Sanjosé S et al (2013) Worldwide human papillomavirus genotype attribution in over 2000 cases of intraepithelial and invasive lesions of the vulva Eur J Cancer 49 528-535 DOI: 10.1016/j.ejca.2013.06.033

10. Van Seters M, van Beurden M and de Craen AJ (2005) Is the assumed natural history of vulvar intraepithelial neoplasia III based on enough evidence? A systematic review of 3322 published patients Gynecol Oncol 97 645-651 DOI: 10.1016/j.ygyno.2005.02.012 PMID: 15863172

11. Jones RW, Rowan DM and Stewart AW (2005) Vulvar intraepithelial neoplasia: aspects of the natural history and outcome in 405 women Obstet Gynecol 106 1319-1326 DOI: 10.1097/01.AOG.0000187301.76283.7f PMID: 16319258

12. Hillemanns $P$ et al (2006) Evaluation of different treatment modalities for vulvar intraepithelial neoplasia (VIN): CO(2) laser vaporization, photodynamic therapy, excision and vulvectomy Gynecol Oncol 100 271-275 DOI: 10.1016/j.ygyno.2005.08.012

13. van de Nieuwenhof HP, van der Avoort IA and de Hullu JA (2008) Review of squamous premalignant vulvar lesions Crit Rev Oncol Hematol 68(2) 131-156 DOI: 10.1016/j.critrevonc.2008.02.012 PMID: 18406622

14. Ho GY et al (1998) Natural history of cervicovaginal papillomavirus infection in young women N Engl J Med $338423-428$ DOI: 10.1056/NEJM199802123380703 PMID: 9459645

15. Bourgault Villada I et al (2004) Spontaneous regression of grade 3 vulvar intraepithelial neoplasia associated with human papillomavirus-16-specific CD4(+) and CD8(+) T-cell responses Cancer Res 64 8761-8766 DOI: 10.1158/0008-5472.CAN-042455 PMID: 15574788

16. Gul N, Ganesan R and Luesley DM (2004) Characterizing T-cell response in low-grade and high-grade vulval intraepithelial neoplasia, study of CD3, CD4 and CD8 expressions Gynecol Oncol 94 48-53 DOI: 10.1016/j.ygyno.2004.02.037 PMID: 15262118

17. Santegoets LA et al (2008) Reduced local immunity in HPV-related VIN: expression of chemokines and involvement of immunocompetent cells Int J Cancer 123 616-622 DOI: 10.1002/ijc.23545 PMID: 18498128

18. Singh $\mathrm{K}$ et al (2006) Antigen processing and correlation with immunological response in vulval intraepithelial neoplasia-a study of CD1a, CD54 and LN3 expression Gynecol Oncol 102 489-492 DOI: 10.1016/j.ygyno.2006.01.036 PMID: 16516282 
19. van Seters $\mathrm{M}$ et al (2008) Disturbed patterns of immunocompetent cells in usual-type vulvar intraepithelial neoplasia Cancer Res 68 6617-6622 DOI: 10.1158/0008-5472.CAN-08-0327 PMID: 18701485

20. Meeuwis KA et al (2010) Skin cancer and (pre)malignancies of the female genital tract in renal transplant recipients Transpl Int 23 191-199 DOI: 10.1111/j.1432-2277.2009.00975.x

21. van Esch EM et al (2012) Treatment failure in patients with HPV 16-induced vulvar intraepithelial neoplasia: understanding different clinical responses to immunotherapy Expert Rev Vaccines 11(7) 821-840 DOI: 10.1586/erv.12.56 PMID: 22913259

22. Ohtani $\mathrm{N}$ et al (2004) The p16INK4a-RB pathway: molecular link between cellular senescence and tumor suppression $J$ Med Invest 51 146-153 DOI: 10.2152/jmi.51.146 PMID: 15460900

23. Rufforny I et al (2005) Human papillomavirus infection and p16(INK4a) protein expression in vulvar intraepithelial neoplasia and invasive squamous cell carcinoma J Low Genit Tract Dis 9 108-113 DOI: 10.1097/00128360-200504000-00007 PMID: 15870532

24. Van der Avoort IA et al (2006) Vulvar squamous cell carcinoma is a multifactorial disease following two separate and independent pathways Int J Gynecol Pathol 25 22-29 DOI: 10.1097/01.pgp.0000177646.38266.6a

25. Hoevenaars BM et al (2008) A panel of p16(INK4A), MIB1 and p53 proteins can distinguish between the 2 pathways leading to vulvar squamous cell carcinoma Int J Cancer 123(12) 2767-2773 DOI: $10.1002 / i j c .23857$ PMID: 18798277

26. Santegoets LA et al (2012) Different DNA damage and cell cycle checkpoint control in low- and high-risk human papillomavirus infections of the vulva Int J Cancer 130 2874-2885 DOI: $\underline{10.1002 / i j c .26345}$

27. Mantovani $F$ and Banks $L$ (2001) The human papillomavirus E6 protein and its contribution to malignant progression Oncogene 20 7874-7887 DOI: 10.1038/sj.onc.1204869 PMID: 11753670

28. Munger $\mathrm{K}$ et al (2001) Biological activities and molecular targets of the human papillomavirus E7 oncoprotein Oncogene 20 7888-7898 DOI: $10.1038 /$ sj.onc.1204860 PMID: 11753671

29. Santos $\mathrm{M}$ et al (2004) Immunohistochemical staining for p16 and p53 in premalignant and malignant epithelial lesions of the vulva Int J Gynecol Pathol 23 206-214 DOI: 10.1097/01.pgp.0000130108.03231.89 PMID: 15213596

30. O'Neill CJ and McCluggage WG (2006) p16 expression in the female genital tract and its value in diagnosis Adv Anat Pathol 13 8-15 DOI: 10.1097/01.pap.0000201828.92719.f3

31. Van de Nieuwenhof HP, van der Avoort IA and de Hullu JA (2008) Review of squamous premalignant vulvar lesions Crit Rev Oncol Hematol 68(2) 131-156 DOI: 10.1016/j.critrevonc.2008.02.012 PMID: 18406622

32. McNally OM et al (2002) VIN 3: a clinicopathologic review Int J Gynecol Cancer 12 490-495 DOI: 10.1046/j.1525-1438.2002.01140.x PMID: 12366668

33. Preti $\mathrm{M}$ et al (2014) Vulvar intraepithelial neoplasia Best Pract Res Clin Obstet Gynaecol 28(7) 1051-1062 DOI: 10.1016/j. bpobgyn.2014.07.010 PMID: 25106700

34. Modesitt SC et al (1998) Vulvar intraepithelial neoplasia III: occult cancer and the impact of margin status on recurrence Obstet Gynecol 92 962-966 DOI: 10.1016/S0029-7844(98)00350-0 PMID: 9840558

35. loffe YJ et al (2013) Low yield of residual vulvar carcinoma and dysplasia upon re-excision for close or positive margins Gynecol Oncol 129 528-532 DOI: 10.1016/j.ygyno.2013.02.033 PMID: 23474347

36. Penna C et al (2002) CO2 laser surgery for vulvar intraepithelial neoplasia. Excisional, destructive and combined techniques J Reprod Med 47 913-918 PMID: 12497680

37. Leufflen $L$ et al (2013) Treatment of vulvar intraepithelial neoplasia with $\mathrm{CO}(2)$ laser vaporization and excision surgery $\mathrm{J}$ Low Genit Tract Dis 17(4) 446-451 DOI: 10.1097/LGT.0b013e318284c1ed PMID: 23609594 
38. Husseinzadeh $N$ and Recinto $C$ (1999) Frequency of invasive cancer in surgically excised vulvar lesions with intraepithelial neoplasia (VIN3) Gynecol Oncol 73 119-120 DOI: 10.1006/gyno.1998.5327 PMID: 10094891

39. Polterauer $S$ et al (2009) Accuracy of preoperative vulva biopsy and the outcome of surgery in vulvar intraepithelial neoplasia 2 and 3 Int J Gynecol Pathol 28 559-562 DOI: 10.1097/PGP.0b013e3181a934d4 PMID: 19851204

40. Powell LC Jr et al (1986) Carcinoma in situ of the vulva. A clinicopathologic study of 50 cases $J$ Reprod Med 31 808-814 PMID: $\underline{3772899}$

41. Beutner KR et al (1998) Imiquimod, a patient-applied immune-response modifier for treatment of external genital warts Antimicrob Agents Chemother 42(4) 789-794 PMID: 9559784 PMCID: 105543

42. Kono $\mathrm{T}$ et al (1994) Effects of a novel topical immunomodulator, imiquimod, on keratinocyte cytokine gene expression Lymphokine Cytokine Res 13(2) 71-76 PMID: 8061117

43. Reiter MJ et al (1994) Cytokine induction in mice by the immunomodulator imiquimod J Leukoc Biol 55(2) 234-240 PMID: $\underline{7507969}$

44. Testerman TL et al (1995) Cytokine induction by the immunomodulators imiquimod and S-27609 J Leukoc Biol 58(3) 365-372 PMID: $\underline{7665993}$

45. Meyer T and Stockfleth E (2008) Clinical investigations of toll-like receptor agonists Expert Opin Investig Drugs 17(7) 1051-1065 DOI: $\underline{10.1517 / 13543784.17 .7 .1051}$ PMID: 18549341

46. Van Seters M et al (2008) Treatment of vulvar intraepithelial neoplasia with topical imiquimod N Engl J Med 358 1465-1473 DOI: 10.1056/NEJMoa072685 PMID: 18385498

47. Terlou A et al (2011) Treatment of vulvar intraepithelial neoplasia with topical imiquimod: seven years median follow-up of a randomized clinical trial Gynecol Oncol 121 157-162 DOI: 10.1016/j.ygyno.2010.12.340 PMID: 21239049

48. Terlou A et al (2010) Imiquimod-induced clearance of HPV is associated with normalization of immune cell counts in usual type vulvar intraepithelial neoplasia Int J Cancer 127(12) 2831-2840 DOI: 10.1002/ijc.25302

49. Pepas L et al (2011) Medical interventions for high grade vulvar intraepithelial neoplasia Cochrane Database Syst Rev (4) CD007924 DOI: 10.1002/14651858.CD007924.pub2

50. Gentile M et al (2014) Adjuvant topical treatment with imiquimod 5\% after excisional surgery for VIN2/3 Eur Rev Med Pharmacol Sci 18(19) 2949-2952 PMID: 25339491

51. Van Esch EM et al (2015) Expression of coinhibitory receptors on T cells in the microenviroment of usual vulvar intraepithelial neoplasia is related to proinflammatory effector T cells and an increased recurrence-free survival Int $J$ Cancer 136(4) E95-E106 DOI: $\underline{10.1002 / i j c .29174}$

52. Terlou A et al (2010) Premalignant epithelial disorders of the vulva: squamous intraepithelial neoplasia, vulvar Paget's disease and melanoma in situ Acta Obstet Gynecol Scand 89(6) 741-748 DOI: 10.3109/00016341003739575 PMID: 20504079

53. Davidson EJ et al (2004) Effect of TA-CIN (HPV 16 L2E6E7) booster immunisation in vulval intraepithelial neoplasia patients previously vaccinated with TA-HPV (vaccinia virus encoding HPV 16/18 E6E7) Vaccine 22 2722-2729 DOI: 10.1016/j. vaccine.2004.01.049 PMID: $\underline{15246603}$

54. Smyth LJ et al (2004) Immunological responses in women with human papillomavirus type 16 (HPV-16)-associated anogenital intraepithelial neoplasia induced by heterologous prime-boost HPV-16 oncogene vaccination Clin Cancer Res $102954-2961$ DOI: $\underline{10.1158 / 1078-0432 . C C R-03-0703}$ PMID: $\underline{15131030}$

55. Kenter GG et al (2009) Vaccination against HPV-16 oncoproteins for vulvar intraepithelial neoplasia N Engl J Med $3611838-$ 1847 DOI: $\underline{10.1056 / N E J M o a 0810097}$ PMID: 19890126 
56. Abdel-Hady ES et al (2001) Immunological and viral factors associated with the response of vulval intraepithelial neoplasia to photodynamic therapy Cancer Res 61 192-196 PMID: 11196160

57. Fehr MK et al (2002) Photodynamic therapy of vulvar and vaginal condyloma and intraepithelial neoplasia using topically applied 5-aminolevulinic acid Lasers Surg Med 30 273-279 DOI: 10.1002/lsm.10048 PMID: 11948597

58. Hillemanns $\mathrm{P}$ et al (2000) Photodynamic therapy of vulvar intraepithelial neoplasia using 5-aminolevulinic acid Int J Cancer 85 649-653 PMID: 10699944

59. Kurwa HA, Barlow RJ and Neill S (2000) Single-episode photodynamic therapy and vulval intraepithelial neoplasia type III resistant to conventional therapy Br J Dermatol 143 1040-1042 DOI: 10.1046/j.1365-2133.2000.03785.x PMID: 11069517

60. Zawislak A et al (2009) Clinical and immunohistochemical assessment of vulval intraepithelial neoplasia following photodynamic therapy using a novel bioadhesive patch-type system loaded with $\mathbf{5}$-aminolevulinic acid Photodiagnosis Photodyn Ther 6 28-40 DOI: 10.1016/j.pdpdt.2009.03.004 PMID: 19447369

61. Stern PL and Einstein MH (2010) From HPV infection to oncogenesis: a brief review of the complex immunobiological events Curr Cancer Ther Rev 6(2) 110-116 DOI: 10.2174/157339410791202565

62. Van der Burg SH and Melief CJ (2011) Therapeutic vaccination against human papilloma virus induced malignancies Curr Opin Immunol 23 252-257 DOI: 10.1016/j.coi.2010.12.010 PMID: 21237632

63. Stern PL et al (2012) Therapy of human papillomavirus-related disease Vaccine 30 Suppl 5 F71-F82 DOI: 10.1016/j. vaccine.2012.05.091 PMID: 23199967 PMCID: 4155500

64. Davidson EJ et al (2003) Immunological and clinical responses in women with vulval intraepithelial neoplasia vaccinated with a vaccinia virus encoding human papillomavirus 16/18 oncoproteins Cancer Res 63 6032-6041 PMID: 14522932

65. Winters $U$ et al (2008) Clinical and immunologic results of a phase II trial of sequential imiquimod and photodynamic therapy for vulval intraepithelial neoplasia Clin Cancer Res 14 5292-5299 DOI: 10.1158/1078-0432.CCR-07-4760 PMID: 18698049

66. Daayana S et al (2010) Phase II trial of imiquimod and HPV therapeutic vaccination in patients with vulval intraepithelial neoplasia Br J Cancer 102 1129-1136 DOI: 10.1038/sj.bjc.6605611 PMID: 20234368 PMCID: 2853099

67. Welters MJ et al (2010) Success or failure of vaccination for HPV16-positive vulvar lesions correlates with kinetics and phenotype of induced T-cell responses Proc Natl Acad Sci USA 107(26) 11895-11899 DOI: 10.1073/pnas.1006500107 PMID: 20547850 PMCID: $\underline{2900675}$

68. Van Poelgeest Ml et al (2005) Detection of human papillomavirus (HPV) 16-specific CD41 T-cell immunity in patients with persistent HPV16-induced vulvar intraepithelial neoplasia in relation to clinical impact of imiquimod treatment Clin Cancer Res 11 5273-5280 DOI: 10.1158/1078-0432.CCR-05-0616 PMID: 16033846

69. Van Esch EM et al (2014) Alterations in classical and nonclassical HLA expression in recurrent and progressive HPV-induced usual vulvar intraepithelial neoplasia and implications for immunotherapy Int J Cancer 135(4) 830-842 DOI: 10.1002/ijc.28713 PMID: 24415578

70. Muñoz $\mathrm{N}$ et al (2010) Impact of human papillomavirus (HPV)-6/11/16/18 vaccine on all HPV-associated genital diseases in young women J Natl Cancer Inst 102 325-339 DOI: 10.1093/jnci/djp534 PMID: 20139221

71. Jemal A et al (2013) Annual report to the nation on the status of cancer, 1975-2009, featuring the burden and trends in human papillomavirus(HPV)-associated cancers and HPV vaccination coverage levels J Natl Cancer Inst 105 175-201 DOI: $10.1093 /$ inci/djs491 PMID: 23297039 PMCID: 3565628

72. Paavonen $\mathrm{J}$ et al (2009) Efficacy of human papillomavirus (HPV)-16/18 AS04-adjuvanted vaccine against cervical infection and precancer caused by oncogenic HPV types (PATRICIA): final analysis of a double-blind, randomised study in young women Lancet 374 301-314 DOI: 10.1016/S0140-6736(09)61248-4 PMID: 19586656 
73. Brown DR et al (2009) The impact of quadrivalent human papillomavirus (HPV; types 6, 11, 16, and 18) L1 virus-like particle vaccine on infection and disease due to oncogenic nonvaccine HPV types in generally HPV-naive women aged 16-26 years J Infect Dis 199 926-935 DOI: 10.1086/597307 PMID: 19236279

74. Einstein $\mathrm{MH}$ et al (2011) Comparison of the immunogenicity of the human papillomavirus (HPV)-16/18 vaccine and the HPV-6/11/16/18 vaccine for oncogenic non-vaccine types HPV-31 and HPV-45 in healthy women aged 18-45 years Hum Vaccin 7(12) 1359-1373 DOI: 10.4161/hv.7.12.18282 PMID: 22048172 PMCID: $\underline{3338933}$

75. Kemp TJ et al (2011) HPV16/18 L1 VLP vaccine induces cross-neutralizing antibodies that may mediate crossprotection Vaccine 29 2011-2014 DOI: 10.1016/j.vaccine.2011.01.001 PMID: 21241731 PMCID: $\underline{3046309}$

76. Joura EA et al (2012) Effect of the human papillomavirus (HPV) quadrivalent vaccine in a subgroup of women with cervical and vulvar disease: retrospective pooled analysis of trial data BMJ 344 e1401 DOI: 10.1136/bmj.e1401 PMID: 22454089 PMCID: $\underline{3314184}$

77. Jones RW, Rowan DM and Stewart AW (2005) Vulvar intraepithelial neoplasia: aspects of the natural history and outcome in 405 women Obstet Gynecol 106(6) 1319-1326 DOI: 10.1097/01.AOG.0000187301.76283.7f PMID: 16319258 